\title{
Reactive Hyperemia Index Associated With Atherosclerotic Cardiovascular Disease Under Treatment for Lifestyle Diseases
}

\author{
Kota Motozato ${ }^{\text {a, b, d, e }}$, Yasunori Suematsu ${ }^{\text {a, e }}$, Kenji Norimatsu ${ }^{\mathrm{a}, \mathrm{b}}$, \\ Takaaki Kusumoto $^{\mathrm{b}}$, Shin-ichiro Miura ${ }^{\mathrm{a}, \mathrm{c}, \mathrm{f}}$
}

\begin{abstract}
Background: Endothelial dysfunction is an early phase of atherosclerosis and causes atherosclerotic cardiovascular disease (ASCVD), but the cutoff reactive hyperemia index (RHI) for identifying ASCVD patients under treatment for lifestyle diseases is unknown.

Methods: Patients who visited Cardiology Section, Izumi General Medical Center, Kagoshima, Japan and were measured RHI using Endo-PAT 2000 during May 2014 and March 2016 were enrolled. We divided them into ASCVD and non-ASCVD groups and investigated the association with RHI between the groups.

Results: ASCVD group included 195 patients and non-ASCVD group included 288 patients. Age, body mass index and the rates of male, hypertension, diabetes, dyslipidemia and chronic kidney disease in the ASCVD group were significantly higher than those in non-ASCVD group. RHI in the ASCVD group (1.65 (1.40 - 1.92)) was significantly lower than that in the non-ASCVD group (1.73 $(1.45-2.24))(P<0.05)$. In multi-regression analysis, RHI (odds ratio: $0.67,95 \%$ confidence interval: $0.45-0.99, \mathrm{P}=0.04$ ) was independently associated with ASCVD after adjusted by age, sex, body mass index, smoking, hypertension, diabetes, dyslipidemia and chronic kidney disease. The cutoff RHI value between the groups was 1.80 .
\end{abstract}

Conclusion: RHI was significantly associated with ASCVD under treatment for lifestyle diseases and the cutoff value was 1.80 .

Manuscript submitted January 25, 2020, accepted February 17, 2020

aDepartment of Cardiology, Fukuoka University School of Medicine, Fukuoka, Japan

bDepartment of Cardiology, Izumi General Medical Center, Kagoshima, Japan 'Department of Cardiology, Fukuoka University Nishijin Hospital, Fukuoka, Japan

dPresent address: Department of Cardiology, Kumamoto University, Kumamoto, Japan

${ }^{\mathrm{e}}$ These authors contributed equally to this manuscript.

${ }^{f}$ Corresponding Author: Shin-ichiro Miura, Department of Cardiology, Fukuoka University School of Medicine, 7-45-1 Nanakuma, Jonan-Ku, Fukuoka 814-0180, Japan.Email: miuras@cis.fukuoka-u.ac.jp

doi: https://doi.org/10.14740/jocmr4100
Keywords: Reactive hyperemia index; Atherosclerotic cardiovascular disease; Lifestyle disease; Endothelial dysfunction; Cutoff value

\section{Introduction}

Endothelial dysfunction is an early phase of atherosclerosis and causes atherosclerotic cardiovascular disease (ASCVD) [1]. Endothelial dysfunction which means systemic/ peripheral endothelial dysfunction is measured in terms of flow-mediated dilation (FMD) or the reactive hyperemia index (RHI) by peripheral arterial tonometry using an EndoPAT $2000^{\circledR}$ device (Itamar Medical, Israel) [2] or the others. FMD has been reported since 1992, and it is a widely used method in clinical research [3]. RHI was reported to be the test for peripheral vascular endothelial function in 2003 [4]. Both methods are based on the same phenomenon which is endothelial-dependent vasodilator function after transient arterial occlusion. RHI and FMD are recommended as endothelial function test in the Japanese Society of Hypertension Guidelines for the Management of Hypertension [5]. The independent prognostic factors for future cardiovascular events of RHI and FMD were similar magnitude by a metaanalysis [6]. While we have previously demonstrated the effectiveness of FMD [7, 8], the RHI value is easy to obtain and more operator-independent [9].

Several studies have shown various associations of RHI in night-time blood pressure variant patients [10], non-alcoholic fatty liver disease patients [11], obstructive sleep apnea patients [12] and non-obstructive coronary artery disease patients [13], in addition to major cardiovascular events [14]. Angiotensin I converting enzyme inhibitor [15-17], dipeptidyl peptidase-4 inhibitors (DPP-4Is) [18], sodiumglucose co-transporter 2 inhibitors [19] and statin therapy [20] have all been shown to improve endothelial function. For the reasons above, the value of RHI changes due to the underlying diseases and medications, and the cutoff value of RHI for ASCVD has not been determined. It is necessary to determine the cutoff value of RHI for ASCVD in clinical situation. 


\section{Materials and Methods}

\section{Study design}

This was a prospective observational study. We asked all patients who visited Cardiology Section, Izumi General Medical Center, Kagoshima, Japan from May 2014 to March 2016 for checking RHI and all of the consecutive patients who were obtained written informed consent were registered. Four hundred ninety-eight consecutive patients were measured RHI using an Endo-PAT $2000^{\circledR}$. We excluded four patients for hemodialysis and 11 patients for unmeasurable data. The remaining 483 patients were included. The study protocol was approved by the Ethics Committee of Izumi General Medical Center (approval number 30).

\section{RHI}

In all patients, RHI was measured in the morning in the fasting state before the intake of medications. Finger probes were placed on the middle finger of each hand. A constant counter pressure was applied and pulsatile volume changes induced pressure alterations in the finger cuff. The change of the arterial blood volume in the distal finger tip caused a change in the measured peripheral arterial tonometry signal. RHI measurements were analyzed with a computerized, automated algorithm to reduce intra- and interobserver variability of peripheral arterial tonometry signal (Endo-PAT $2000^{\circledR}$ software, Itamar Medical) [14].

\section{Evaluation of ASCVD risk factors}

ASCVD was defined as a past history of ischemic heart disease, cerebrovascular disease, and/or peripheral artery disease. Age, gender, body mass index (BMI) and smoking status (past and current smoker) were evaluated. BMI was calculated as weight $(\mathrm{kg}) /$ height $(\mathrm{m})^{2}$. The histories of hypertension (HT), diabetes mellitus (DM), dyslipidemia (DLP) and chronic kidney disease (CKD) were obtained from medical records. Patients who had a current systolic blood pressure/diastolic blood pressure $\geq 140 / 90 \mathrm{~mm} \mathrm{Hg}$ or who were receiving antihypertensive therapy were considered to have HT. DM was defined using the American Diabetes Association criteria [21]. Patients with low-density lipoprotein cholesterol $\geq 140 \mathrm{mg} /$ $\mathrm{dL}$, triglyceride $\geq 150 \mathrm{mg} / \mathrm{dL}$ and/or high-density lipoprotein cholesterol $<40 \mathrm{mg} / \mathrm{dL}$, or who were receiving lipid-lowering therapy, were considered to have DLP. CKD was defined as an estimated glomerular filtration rate (eGFR) $<60 \mathrm{~mL} /$ $\min / 1.73 \mathrm{~m}^{2}$. EGFR was determined using the abbreviated equation that the Japanese Society of Nephrology modified for Japanese based on the Modification of Diet in Renal Disease study: $194 \times(\text { age }(\text { years }))^{-0.287} \times($ serum creatinine $(\mathrm{mg} /$

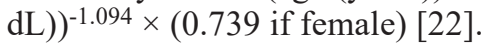

Medication use, including angiotensin II receptor blocker/angiotensin converting enzyme inhibitor, calcium channel blocker, $\beta$-blocker, other anti-hypertension drugs, statin, eicosapentaenoic acid, other anti-dyslipidemia drugs, DPP-4I and other anti-diabetic drugs was evaluated. Serum levels of white blood cell and C-reactive protein were evaluated.

\section{Statistical analyses}

All of the data analyses were performed using the SAS (Statistical Analysis System) Software Package (Ver. 9.4, SAS Institute Inc., Cary, NC, USA) at Fukuoka University (Fukuoka, Japan). Continuous variables with a normal distribution are expressed as mean \pm standard deviation and differences between groups were compared by Student's $t$-test. Continuous variables with a non-normal distribution are expressed as median (interquartile range) and differences between groups were compared by the Wilcoxon rank-sum test. Differences in categorical variables between groups were compared by a Chisquare analysis. Multivariate analysis was performed using a logistic regression analysis for independent variables that were related to the presence of ASCVD, using risk factors including RHI, sex, age, BMI, smoking, HT, DM, DLP and CKD. A receiver-operating characteristic curve analysis was used to determine the cutoff value of RHI for ASCVD at the highest possible sensitivity and specificity levels. A value of $\mathrm{P}<0.05$ was considered significant.

\section{Results}

\section{Baseline patient characteristics}

Table 1 shows the patient characteristics. Age, the percentage of male and BMI were $69(62-76)$ years, $55 \%(\mathrm{n}=265)$ and $24(22-26) \mathrm{kg} / \mathrm{m}^{2}$, respectively. Age, the percentage of male, $\mathrm{BMI}$ and the percentage of smoking in the ASCVD group were significantly higher than those in the non-ASCVD group. The percentages of HT, DM and DLP were $73.9 \%(n=357), 24 \%$ $(\mathrm{n}=116)$ and $75.8 \%(\mathrm{n}=366)$, respectively. The percentages of metabolic syndrome (MetS), HT, DM and DLP in the ASCVD group were significantly higher than those in the nonASCVD group. Inflammation markers such as white blood cell and C-reactive protein in the ASCVD group were significantly higher than those in the non-ASCVD group.

Table 2 shows medication use in the study cohort. Angiotensin II receptor blocker/angiotensin converting enzyme inhibitor, DPP-4I and statin were administered significantly more often in the ASCVD group than in the non-ASCVD group.

\section{RHI}

RHI in all patients, ASCVD group and non-ASCVD group is shown in Figure 1. RHI in the ASCVD group (1.65 (1.40 - 1.92)) was significantly lower than that in the non-ASCVD group $(1.73(1.45-2.24))(\mathrm{P}=0.004)$, although angiotensin II receptor blocker/angiotensin converting enzyme inhibitor, DPP- 
Table 1. Patient Characteristics

\begin{tabular}{lllll}
\hline Variables & All $(\mathbf{n}=\mathbf{4 8 3})$ & ASCVD $(\mathbf{n}=\mathbf{1 9 5})$ & Non-ASCVD $(\mathbf{n}=\mathbf{2 8 8})$ & P value \\
\hline Age, years & $69(62-76)$ & $72(65-77)$ & $67(61-75)$ & $<0.001$ \\
Male, $\mathrm{n}(\%)$ & $265(55)$ & $147(75)$ & $118(41)$ & $<0.001$ \\
BMI, $\mathrm{kg} / \mathrm{m}^{2}$ & $24(22-26)$ & $24.3 \pm 3.1$ & $24(22-26)$ & 0.047 \\
Smoking, n (\%) & $208(43)$ & $124(64)$ & $84(29)$ & $<0.001$ \\
HT, n $(\%)$ & $357(73.9)$ & $171(87.7)$ & $186(64.6)$ & $<0.001$ \\
DM, n $(\%)$ & $116(24)$ & $61(31.3)$ & $55(19.1)$ & 0.003 \\
DLP, n $(\%)$ & $366(75.8)$ & $170(87.2)$ & $196(68.1)$ & $<0.001$ \\
MetS, n $(\%)$ & $172(35.6)$ & $102(52.3)$ & $70(24.3)$ & $<0.001$ \\
WBC, $10^{3} / \mu \mathrm{L}$ & $5,400(4,500-6,700)$ & $5,900(4,800-7,100)$ & $5,200(4,400-6,300)$ & $<0.001$ \\
CRP, $\mathrm{mg} / \mathrm{dL}$ & $0.06(0.03-0.12)$ & $0.07(0.04-0.16)$ & $0.05(0.03-0.10)$ & 0.003 \\
\hline
\end{tabular}

$P$ value shows the comparison with ASCVD vs. non-ASCVD. ASCVD: atherosclerotic cardiovascular disease; BMI: body mass index; HT: hypertension; DM: diabetes mellitus; DLP: dyslipidemia; MetS: metabolic syndrome; WBC: white blood cell; CRP: C-reactive protein.

4I and statin were more administered in the ASCVD group.

\section{Independent risk factors for ASCVD}

A multivariate analysis was performed to identify independent risk factors for ASCVD (Fig. 2). RHI (odds ratio (OR): 0.67, 95\% confidence interval (CI): $0.45-0.99, \mathrm{P}=0.04)$ ), age (OR: 1.07, 95\% CI: $1.04-1.09, \mathrm{P}<0.001$ ), male (OR: $3.36,95 \%$ CI: 1.91 - 5.90, P<0.001), smoking (OR: $2.48,95 \%$ CI: 1.43 4.29, $\mathrm{P}=0.001)$, HT (OR: 2.62, 95\% CI: $1.52-4.50, \mathrm{P}<0.001)$ and DLP (OR: 4.77, 95\% CI: 2.66 - 8.58, P < 0.001) were independently associated with ASCVD. BMI (OR: 0.99, 95\% CI: $0.92-1.06, \mathrm{P}=0.8), \mathrm{DM}(\mathrm{OR}: 1.33,95 \% \mathrm{CI}: 0.80-2.22, \mathrm{P}=$ 0.3 ) and CKD (OR: 0.94, 95\% CI: $0.53-1.68, \mathrm{P}=0.8)$ were not significantly associated with ASCVD. A receiver-operating characteristic curve analysis was performed to determine the optimal cutoff value of RHI for ASCVD under treatment for lifestyle diseases (Fig. 3). The cutoff value of RHI was 1.80 and an area under the curve, sensitivity and specificity were $0.58,0.45$ and 0.67 , respectively.

\section{Discussion}

We investigated the cutoff point of RHI for ASCVD in clinical patients under treatment for lifestyle diseases. RHI, age, male, smoking, HT and DLP were independently associated with ASCVD. The cutoff point of RHI for ASCVD was 1.80.

RHI measures digital reactive hyperemia, which is partially mediated by endothelium-derived nitric oxide and reflects endothelial dysfunction [23], and is associated with ASCVD [14]. Rubinshtein et al reported that RHI has a similar predictive power to FMD in patients with diagnosed obstructive coronary artery disease [14]. In Japanese Guideline for Non-Invasive Vascular Function Test which was es-

Table 2. Medications

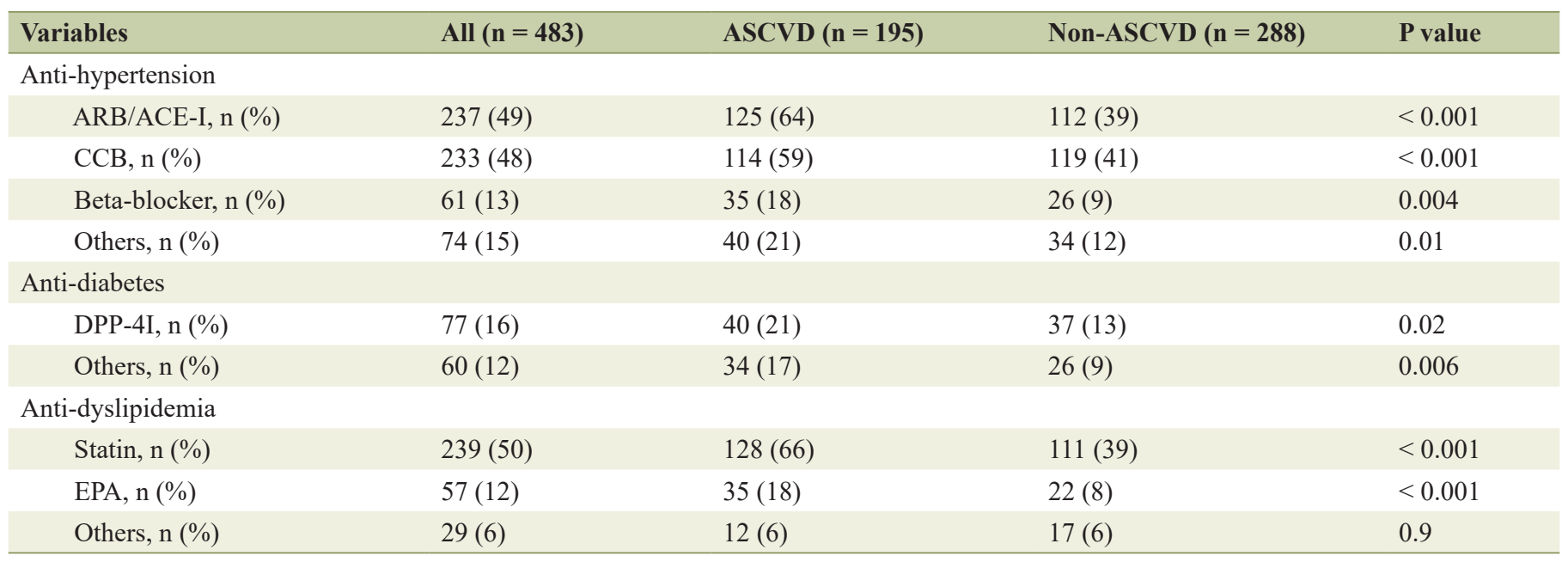

$P$ value shows the comparison with ASCVD vs. non-ASCVD. ASCVD: atherosclerotic cardiovascular disease; ARB: angiotensin II receptor blocker; ACE-I: angiotensin converting enzyme inhibitor; CCB: calcium channel blocker; EPA: eicosapentaenoic acid; DPP-4I: dipeptidyl peptidase-4 inhibitor. 


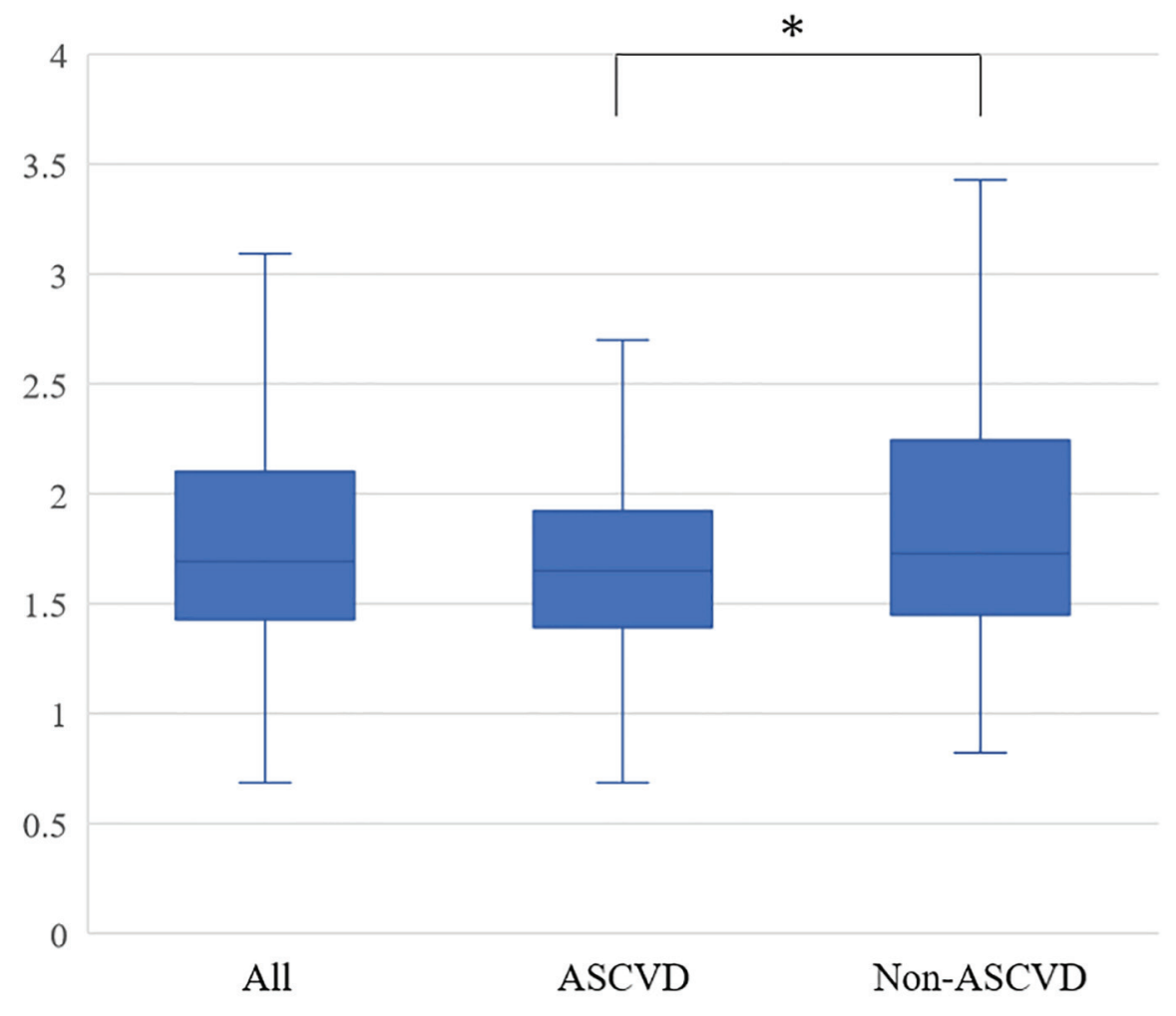

Figure 1. Reactive hyperemia indexes in all patients, the ASCVD and non-ASCVD groups. ASCVD: atherosclerotic cardiovascular disease. ${ }^{*} \mathrm{P}<0.05$

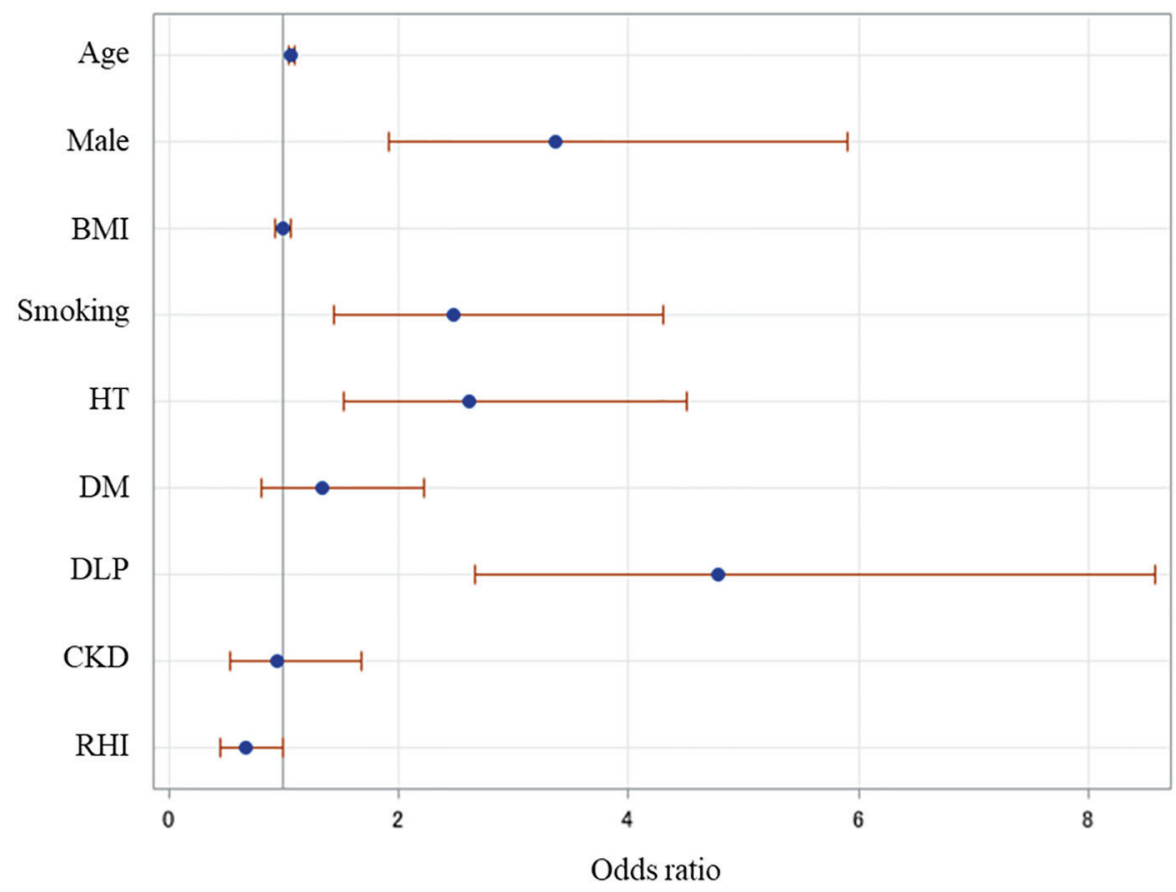

Figure 2. Independent risk factors for ASCVD. A multiple logistic regression analysis was performed. Age, RHI, male, smoking, HT and DLP were independent factors associated with ASCVD. ASCVD: atherosclerotic cardiovascular disease; BMI: body mass index; RHI: reactive hyperemia index; HT: hypertension; DM: diabetes mellitus; DLP: dyslipidemia; CKD: chronic kidney disease. 


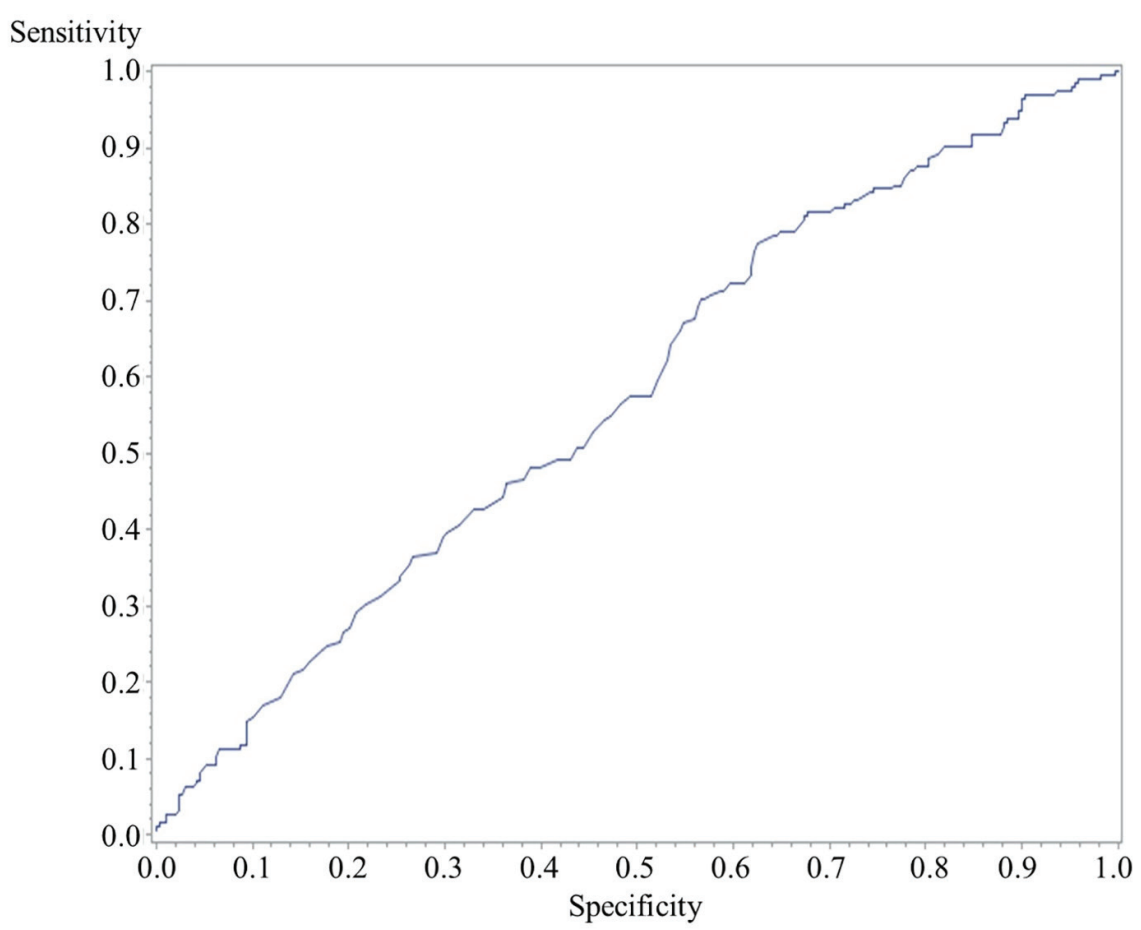

Figure 3. A receiver-operating characteristic curve of the cutoff value of RHI for ASCVD. The cutoff value of RHI was 1.80 and an area under the curve, sensitivity and specificity were $0.58,0.45$ and 0.67 , respectively. RHI: reactive hyperemia index; ASCVD: atherosclerotic cardiovascular disease.

tablished by collaborated 10 societies including The Japanese Circulation Society, the Japanese Society of Hypertension and Japanese College of Cardiology, the cutoff value of RHI for endovascular injury was recommended less than 1.67, based on a clinical study [24]. The cutoff value of RHI for ASCVD is usually lower than that for endovascular injury, because ASCVD has progressed pathophysiology of endovascular injury. But our study showed the cutoff value of RHI for ASCVD was higher than 1.67. It would cause medications including angiotensin converting enzyme inhibitor, DPP-4I and statin for improving the RHI. Matsuzawa et al reported that the cutoff value of RHI for detecting obstructive coronary artery disease and non-obstructive coronary artery disease in women was 1.82 [25]. Tanaka et al reported that the border zone of RHI for endothelial dysfunction was $1.67-2.10$ [2]. This result suggests that we should care for endovascular injury when the value of RHI is still high in clinical situation.

MetS, HT, DM, DLP and CKD are common risk factors of ASCVD [26-29]. In this study, the percentages of these underlying diseases and the use of anti-hypertensive, anti-diabetic and anti-dyslipidemia medications in the ASCVD group were higher than those in the non-ASCVD group. Sodium-glucose co-transporter 2 inhibitor was not administered in this cohort because sodium-glucose co-transporter 2 inhibitor was not widely used in the study period yet. Inflammation leads to the development of atherosclerosis and causes ASCVD [30, 31]. In this study, white blood cell and C-reactive protein in the ASCVD group were significantly higher than those in the non-ASCVD group.

This study has several limitations. We defined ASCVD as a past history of ischemic heart disease, cardiovascular disease and/or peripheral artery disease, but we did not confirm the diagnosis by examinations. Further clinical studies will be needed to confirm the results of our study.

In conclusion, age, RHI, male, smoking, HT and DLP were independently associated with ASCVD and the cutoff point of RHI for ASCVD was 1.80 .

\section{Acknowledgments}

We are deeply grateful to all medical staff at Izumi General Medical Center, Kagoshima, Japan.

\section{Financial Disclosure}

None to declare.

\section{Conflict of Interest}

We have no conflict of interest.

\section{Informed Consent}

Written informed consent was obtained. 


\section{Author Contributions}

TK and SM contributed to the conception or design of the work. KM, YS and KN contributed to the acquisition, analysis, or interpretation of data for the work. KM and YS drafted the manuscript. TK and SM critically revised the manuscript. All gave final approval and agree to be accountable for all aspects of work ensuring integrity and accuracy.

\section{Data Availability}

Any inquiries regarding supporting data availability of this study should be directed to the corresponding author.

\section{References}

1. Widlansky ME, Gokce N, Keaney JF, Jr., Vita JA. The clinical implications of endothelial dysfunction. J Am Coll Cardiol. 2003;42(7):1149-1160.

2. Tanaka A, Tomiyama H, Maruhashi T, Matsuzawa Y, Miyoshi T, Kabutoya T, Kario K, et al. Physiological Diagnostic Criteria for Vascular Failure. Hypertension. 2018;72(5):1060-1071.

3. Celermajer DS, Sorensen KE, Gooch VM, Spiegelhalter DJ, Miller OI, Sullivan ID, Lloyd JK, et al. Non-invasive detection of endothelial dysfunction in children and adults at risk of atherosclerosis. Lancet. 1992;340(8828):11111115.

4. Kuvin JT, Patel AR, Sliney KA, Pandian NG, Sheffy J, Schnall RP, Karas RH, et al. Assessment of peripheral vascular endothelial function with finger arterial pulse wave amplitude. Am Heart J. 2003;146(1):168-174.

5. Umemura S, Arima H, Arima S, Asayama K, Dohi Y, Hirooka Y, Horio T, et al. The Japanese society of hypertension guidelines for the management of hypertension (JSH 2019). Hypertens Res. 2019;42(9):1235-1481.

6. Matsuzawa Y, Kwon TG, Lennon RJ, Lerman LO, Lerman A. Prognostic value of flow-mediated vasodilation in brachial artery and fingertip artery for cardiovascular events: a systematic review and meta-analysis. J Am Heart Assoc. 2015;4(11):e002270.

7. Koyoshi R, Hitaka-Yoshimine Y, Shiga Y, Kuwano T, Sugihara M, Ike A, Iwata A, et al. Associations between microalbuminuria and parameters of flow-mediated vasodilatation obtained by continuous measurement approaches. Clin Exp Hypertens. 2018;40(8):715-720.

8. Koyoshi R, Miura S, Kumagai N, Shiga Y, Mitsutake R, Saku K. Clinical significance of flow-mediated dilation, brachial intima-media thickness and pulse wave velocity in patients with and without coronary artery disease. Circ J. 2012;76(6):1469-1475.

9. Liu W, Meng M, Chen J, Wang L, Sun Z, Li X, Zhou J, et al. Reactive Hyperemia Index in Patients on Maintenance Hemodialysis: Cross-sectional Data from a Cohort Study. Sci Rep. 2017;7:45757.

10. Saito Y, Kitahara H, Nakayama T, Fujimoto Y, Kobayashi
Y. Night-time blood pressure variability negatively correlated with reactive hyperemia index. Int J Cardiol. 2017;230:332-334.

11. Tuttolomondo A, Petta S, Casuccio A, Maida C, Corte VD, Daidone M, Di Raimondo D, et al. Reactive hyperemia index (RHI) and cognitive performance indexes are associated with histologic markers of liver disease in subjects with non-alcoholic fatty liver disease (NAFLD): a case control study. Cardiovasc Diabetol. 2018;17(1):28.

12. Shpilsky D, Erqou S, Patel SR, Kip KE, Ajala O, Aiyer A, Strollo PJ, et al. Association of obstructive sleep apnea with microvascular endothelial dysfunction and subclinical coronary artery disease in a community-based population. Vasc Med. 2018;23(4):331-339.

13. Michelsen MM, Mygind ND, Pena A, Aziz A, Frestad D, Host N, Prescott E, et al. Peripheral reactive hyperemia index and coronary microvascular function in women with no obstructive CAD: the iPOWER study. JACC Cardiovasc Imaging. 2016;9(4):411-417.

14. Rubinshtein R, Kuvin JT, Soffler M, Lennon RJ, Lavi S, Nelson RE, Pumper GM, et al. Assessment of endothelial function by non-invasive peripheral arterial tonometry predicts late cardiovascular adverse events. Eur Heart J. 2010;31(9):1142-1148.

15. Okuro M, Morimoto S, Takahashi T, Okaishi K, Nakahashi T, Murai H, Iwai K, et al. Angiotensin I-converting enzyme inhibitor improves reactive hyperemia in elderly hypertensives with arteriosclerosis obliterans. Hypertens Res. 2006;29(9):655-663.

16. Higashi Y, Sasaki S, Nakagawa K, Ueda T, Yoshimizu A, Kurisu S, Matsuura H, et al. A comparison of angiotensinconverting enzyme inhibitors, calcium antagonists, betablockers and diuretic agents on reactive hyperemia in patients with essential hypertension: a multicenter study. J Am Coll Cardiol. 2000;35(2):284-291.

17. Ki YJ, Seo JB, Kim HL, Lim WH, Seo HY, Lee JY, Chung WY. Comparison of endothelial function improvement estimated with reactive hyperemia index between ramipril and telmisartan in hypertensive patients. Clin Hypertens. 2017;23:4.

18. Koyama T, Tanaka A, Yoshida H, Oyama JI, Toyoda S, Sakuma M, Inoue T, et al. Comparison of the effects of linagliptin and voglibose on endothelial function in patients with type 2 diabetes and coronary artery disease: a prospective, randomized, pilot study (EFFORT). Heart Vessels. 2018;33(8):958-964.

19. Sugiyama S, Jinnouchi H, Kurinami N, Hieshima K, Yoshida A, Jinnouchi K, Nishimura H, et al. The SGLT2 inhibitor dapagliflozin significantly improves the peripheral microvascular endothelial function in patients with uncontrolled type 2 diabetes mellitus. Intern Med. 2018;57(15):2147-2156.

20. Takayama T, Hiro T, Yoda S, Fukamachi D, Haruta H, Kogo T, Mineki T, et al. Effect of Aggressive lipid-lowering treatment with Rosuvastatin on vascular endoTHelium function: evaluation of vascular endothelium function (EARTH study). Heart Vessels. 2018;33(6):590-594.

21. American Diabetes A. 2. Classification and Diagnosis of Diabetes: Standards of Medical Care in Diabetes-2018. 
Diabetes Care. 2018;41(Suppl 1):S13-S27.

22. Matsuo S, Imai E, Horio M, Yasuda Y, Tomita K, Nitta $\mathrm{K}$, Yamagata $\mathrm{K}$, et al. Revised equations for estimated GFR from serum creatinine in Japan. Am J Kidney Dis. 2009;53(6):982-992.

23. Nohria A, Gerhard-Herman M, Creager MA, Hurley S, Mitra D, Ganz P. Role of nitric oxide in the regulation of digital pulse volume amplitude in humans. J Appl Physiol (1985). 2006;101(2):545-548.

24. Bonetti PO, Barsness GW, Keelan PC, Schnell TI, Pumper GM, Kuvin JT, Schnall RP, et al. Enhanced external counterpulsation improves endothelial function in patients with symptomatic coronary artery disease. J Am Coll Cardiol. 2003;41(10):1761-1768.

25. Matsuzawa Y, Sugiyama S, Sugamura K, Nozaki T, Ohba K, Konishi M, Matsubara J, et al. Digital assessment of endothelial function and ischemic heart disease in women. J Am Coll Cardiol. 2010;55(16):1688-1696.

26. Stone NJ, Robinson JG, Lichtenstein AH, Bairey Merz CN, Blum CB, Eckel RH, Goldberg AC, et al. 2013 ACC/ AHA guideline on the treatment of blood cholesterol to reduce atherosclerotic cardiovascular risk in adults: a report of the American College of Cardiology/American
Heart Association Task Force on Practice Guidelines. Circulation. 2014;129(25 Suppl 2):S1-45.

27. Andrus B, Lacaille D. 2013 ACC/AHA guideline on the assessment of cardiovascular risk. J Am Coll Cardiol. 2014;63(25 Pt A):2886.

28. Verdecchia P, Dagenais G, Healey J, Gao P, Dans AL, Chazova I, Binbrek AS, et al. Blood pressure and other determinants of new-onset atrial fibrillation in patients at high cardiovascular risk in the ongoing telmisartan alone and in combination with ramipril global endpoint trial/telmisartan randomized assessment study in ACE intolerant subjects with cardiovascular disease studies. J Hypertens. 2012;30(5):1004-1014.

29. Tonelli M, Karumanchi SA, Thadhani R. Epidemiology and Mechanisms of Uremia-Related Cardiovascular Disease. Circulation. 2016;133(5):518-536.

30. Pant S, Deshmukh A, Gurumurthy GS, Pothineni NV, Watts TE, Romeo F, Mehta JL. Inflammation and atherosclerosis - revisited. J Cardiovasc Pharmacol Ther. 2014;19(2):170-178.

31. Lucas AR, Korol R, Pepine CJ. Inflammation in atherosclerosis: some thoughts about acute coronary syndromes. Circulation. 2006;113(17):e728-732. 